\title{
Acalabrutinib Regimen
}

National Cancer Institute

\section{Source}

National Cancer Institute. Acalabrutinib Regimen. NCI Thesaurus. Code C159981.

A chemotherapy regimen consisting of acalabrutinib that may be used in the treatment

of mantle cell lymphoma (MCL), chronic lymphocytic leukemia (CLL) and small lymphocytic lymphoma (SLL). 